\title{
Urea in Dermatology: A Review of its Emollient, Moisturizing, Keratolytic, Skin Barrier Enhancing and Antimicrobial Properties
}

\author{
Jaime Piquero-Casals (D) - Daniel Morgado-Carrasco • Corinne Granger • \\ Carles Trullàs · América Jesús-Silva · Jean Krutmann
}

Received: July 24, 2021 / Published online: October 1, 2021

(C) The Author(s) 2021

\begin{abstract}
Urea is a hygroscopic molecule (capable of absorbing water) present in the epidermis as a component of the natural moisturizing factor (NMF) and is essential for the adequate hydration and integrity of the stratum corneum. Urea improves skin barrier function including antimicrobial defense by regulating gene expression in keratinocytes relevant for their differentiation and antimicrobial peptide production. It also plays a fundamental role in regulating keratinocyte proliferation. One of the first uses of urea in modern medicine was the topical treatment of wounds due to its
\end{abstract}

J. Piquero-Casals $(\bowtie) \cdot$ A. Jesús-Silva

Dermik. Multidisciplinary Dermatology Clinic, Barcelona, Spain

e-mail: jaime.piquero@e-campus.uab.cat

D. Morgado-Carrasco

Dermatology Department, Hospital Clínic de

Barcelona, Universitat de Barcelona, Barcelona, Spain

C. Granger · C. Trullàs

Innovation and Development, ISDIN, Barcelona, Spain

J. Krutmann

IUF-Leibniz Research Institute for Environmental

Medicine, Dusseldorf, Germany

J. Krutmann

Medical Faculty, Heinrich Heine University,

Düsseldorf, Germany proteolytic and antibacterial properties. At present, urea is widely used in dermatology to improve skin barrier function and as one of the most common moisturizers and keratolytic agents. Urea-containing formulations are available in diverse formulations and concentrations. Multiple clinical trials on the use of ureacontaining formulations have shown significant clinical improvement in many of the dermatosis presenting with scaly and dry skin such as atopic dermatitis, ichthyosis, xerosis, seborrheic dermatitis and psoriasis, among others. Furthermore, urea can increase skin penetration and optimize the action of topical drugs. Ureabased products are well tolerated; their side effects are mild and are more frequent at high concentration. Here, we present a review of the use of urea in dermatology, discussing its mechanism of action, safety profile and frequent indications.

Keywords: Urea; Keratinocytes; Atopic dermatitis; Ichthyosis; Psoriasis 


\section{Key Summary Points}

Urea is widely used in dermatology to improve skin barrier function and as one of the most common moisturizers and keratolytic agents

Urea plays a fundamental role in regulating keratinocyte proliferation, the skin's barrier function and antimicrobial defense

Urea induces the expression of filaggrin, loricrin and transglutaminase-1, i.e., genes which are important for keratinocyte differentiation and thus skin barrier function

Urea-containing formulations have shown significant clinical improvement in many of the dermatoses presenting with scaly and dry skin such as atopic dermatitis, ichthyosis, xerosis, seborrheic dermatitis and psoriasis

It can be easily incorporated into dermatological preparations because it is highly water soluble

Urea can increase skin and nail penetration and optimize the action of topical drugs

Compounds with low urea concentration (2\%-10\%) are indicated for moisturizing and optimizing the skin's barrier function, medium concentration (10\%-30\%) for moisturizers and keratolytics and high concentrations ( $\geq 30 \%$ ) for keratolytics and debriding necrotic tissue

\section{INTRODUCTION}

Urea is a low-molecular-weight organic molecule composed of a carbonyl group attached to two amine residues. It is produced mainly after hepatic digestion of proteins. Urea is a hygroscopic molecule (capable of absorbing water) present in the epidermis as a component of the natural moisturizing factor (NMF) and is essential for the adequate hydration and integrity of the stratum corneum. A reduction in the hygroscopic capacity of the skin can increase transepidermal water loss (TEWL), deregulate epidermal proliferation and inhibit skin desquamation, inducing hyperkeratosis and pruritus [1].

Urea is a topical emollient and moisturizing, keratolytic and antipruritic agent, and it plays a key role in keratinocyte gene regulation. In Asia, Africa and Europe, the therapeutic use of urine (urotherapy) has been documented for centuries. One of the first uses of urea in modern medicine was the topical treatment of wounds due to its proteolytic and antibacterial properties [2]. Currently, urea is widely used in dermatology. It is prescribed for the treatment of diverse dermatoses presenting with scaly and dry skin such as atopic dermatitis, ichthyosis, seborrheic dermatitis and psoriasis, among others [3]. Furthermore, urea can increase skin penetration and optimize the action of topical drugs [3]. Urea-containing formulations are available in diverse formulations and concentrations.

Topical urea is a well-tolerated treatment; its side effects are mild and are more frequent at high concentration. Here, we present a review of topical urea in dermatology, discussing its mechanism of action, safety profile, formulations and frequent indications.

This article is based on previously conducted studies and does not contain any new studies with human participants or animals performed by any of the authors.

\section{SAFETY PROFILE}

Urea-based formulations are well tolerated. Urea-containing creams may present an unpleasant odor, caused by volatile amine, but it dissipates rapidly [4]. Mild skin irritation is occasionally observed and can be more frequent with use at higher concentration. This adverse effect is temporary and usually self-resolving. We have only found one reported case of allergic contact dermatitis to a cream containing a 
Table 1 Effects of urea on the skin

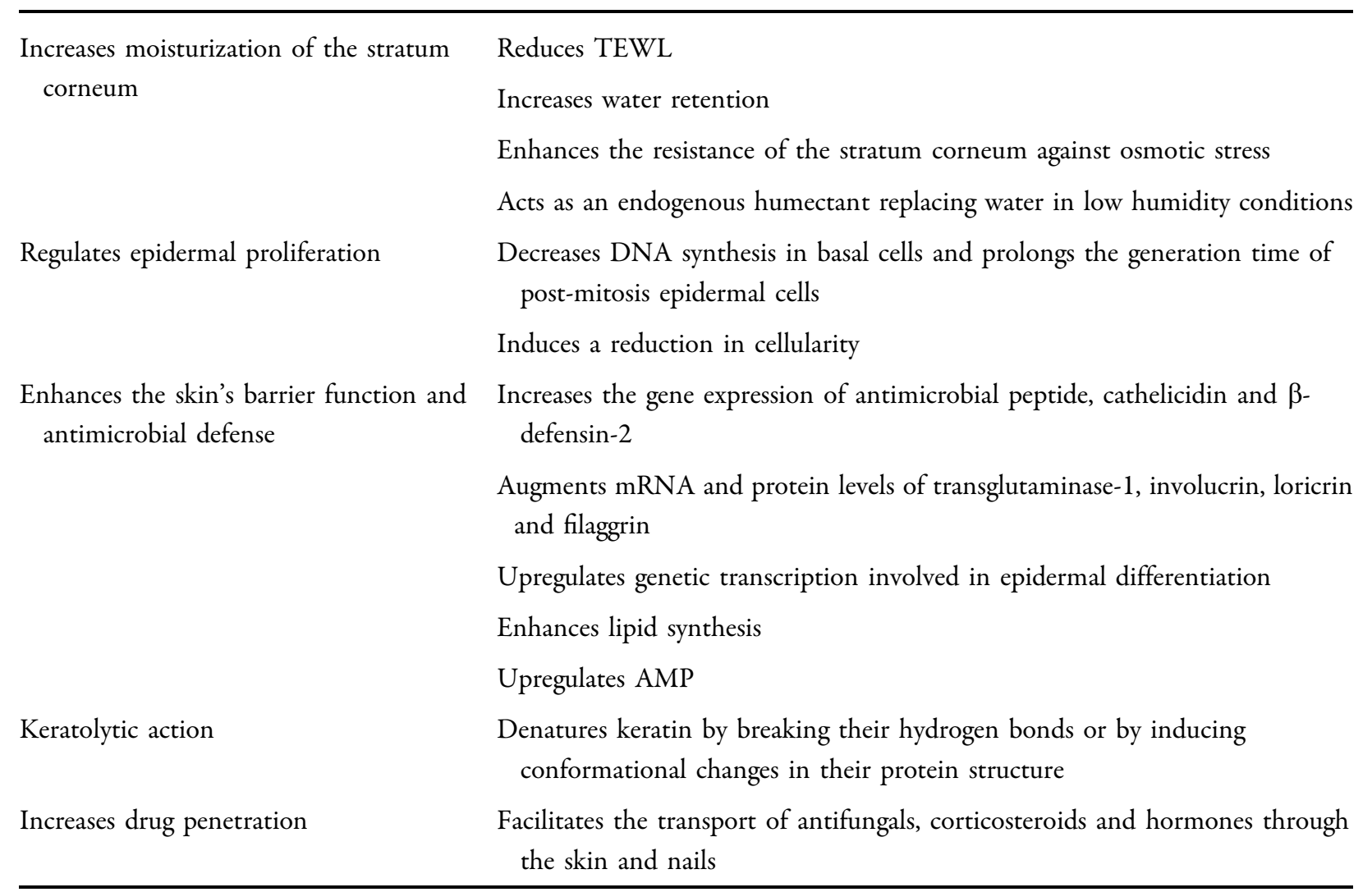

TEWL transepidermal water loss

high concentration of urea, among other components, and the adverse effect was probably secondary to an ingredient other than urea [5].

\section{MECHANISM OF ACTION}

1. Increases moisturization of the stratum corneum (Table 1).

Urea reduces TEWL and increases water retention and the resistance of the stratum corneum against osmotic stress. Furthermore, urea can act as an endogenous humectant replacing water in low humidity conditions $[6,7]$.

2. Improves skin's barrier function and antimicrobial defense (Fig. 1):

Keratinocytes express functionally active urea transporters. In this cell type, urea uptake was found to be associated with active regulation of the transcriptional expression of genes encoding for urea transporters and beyond. Specifically, urea induces the expression of filaggrin, loricrin and transglutaminase-1, i.e., genes which are important for keratinocyte differentiation and thus skin barrier function [8]. Transglutaminase- 1 catalyzes the formation of isopeptide protein-protein crosslinks, allowing the construction of the mechanically resistant envelope composed of proteins such as involucrin and locrinin (its major component), which encloses corneocytes [8]. Filaggrin is a crucial epidermal protein which contributes to the structure of the stratum corneum and to skin barrier function. Filaggrin mutations can be observed in genodermatoses such as ichthyosis vulgaris, caused by loss-of-function mutations in the filaggrin gene, and characterized by dry skin and scaling, or in inflammatory chronic dermatoses such as atopic dermatitis 


\section{MECHANISM OF ACTION OF UREA IN THE SKIN}

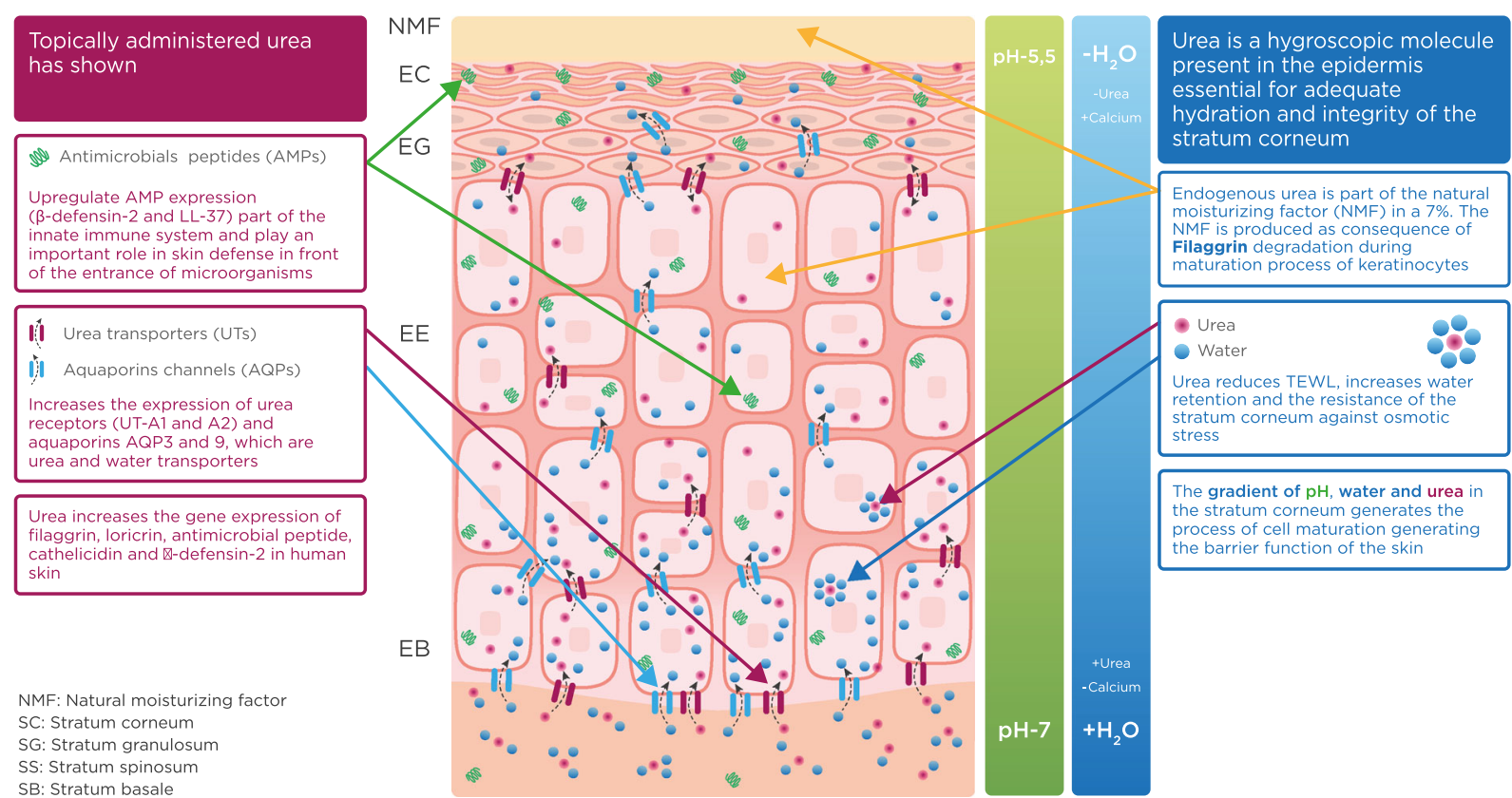

transport is mediated through specific transporters (UT-A1, UT-A2, aquaporin-3 and aquaporin-9). Urea upregulates these transporters and gene expression involved in keratinocyte differentiation, lipid synthesis and antimicrobial peptide production, thus enhancing skin barrier function and antimicrobial defense. Normalize the barrier function of the skin.

Fig. 1 Mechanism of action of urea on the skin. Urea transport is mediated through specific transporters (UTA1, UT-A2, aquaporin-3 and aquaporin-9). Urea upregulates these transporters and gene expression involved in

(AD) [6]. AD patients with filaggrin mutations have more frequent hospital admissions and reduced health-related quality of life than $\mathrm{AD}$ individuals with wild-type fillagrin [6]. Filaggrin expression has shown the strongest upregulation after urea treatment [8]. This is in line with the observation that urea treatment was found to normalize skin barrier function and to upregulate AMP expression in a murine model of $\mathrm{AD}$ [8]. Accordingly, controlled clinical studies have demonstrated that topical application of urea is effective in reducing TEWL and thus in improving skin barrier function. Urea also induces the transcriptional expression of the antimicrobial peptides cathelicidin and $\beta$ defensin-2 in cultured keratinocytes in vitro and in human skin in vivo, further emphasizing its effectiveness in improving skin barrier function [8].

3. Regulates epidermal proliferation keratinocyte differentiation, lipid synthesis and antimicrobial peptide production, thus enhancing skin barrier function and antimicrobial defense

Urea is a highly active molecule and an important regulator of epidermal structure and function. Urea plays a fundamental role in controlling keratinocyte proliferation: it decreases DNA synthesis in basal cells and prolongs the generation time of post-mitosis epidermal cells. Urea induces a reduction in cellularity, resulting in epidermal thinning [9].

\section{Keratolytic action}

Urea at higher concentrations can denature proteins such as keratin by breaking their hydrogen bonds [6] or by inducing conformational changes in their protein structure [3].

\section{Increases drug penetration}

Urea can facilitate the transport of molecules such as antifungals, corticosteroids and hormones through the skin and nails. This effect seems to be secondary to increased water 
absorption by keratinocytes in the presence of urea and to its keratolytic action [6].

\section{TOPICAL FORMULATIONS CONTAINING UREA}

Urea can be easily incorporated into dermatological preparations because it is highly water soluble. There are multiple vehicles and formulations (creams, lotions, emulsions, ointments, gels, shampoos and nail lacquers, among others) and concentrations of urea available in over-the-counter products and prescription medications, which allows great versatility in its prescription. Diverse factors must be considered before choosing the most adequate product: the disorder to be treated, severity, area of the body involved (and its extension) and cosmetic acceptability [10]. In general, compounds with low urea concentration (2\%-10\%) are indicated for moisturizing and optimizing the skin's barrier function [11], medium concentration (10\%-30\%) for moisturizers and keratolytics [12] and high concentrations ( $\geq 30 \%)$ for keratolytics and for debriding necrotic tissue [4] (Table 2).

\section{MAIN INDICATIONS FOR UREA IN DERMATOLOGY}

\author{
Maintaining Healthy Skin
}

Products with low or medium concentration (2\%-20\%) of urea may be indicated for routine skin care in individuals without skin disorders [3, 11-13]. Urea can decrease TEWL [13], increase the water content of the stratum corneum, optimize the skin barrier function and upregulate antimicrobial peptides in the skin [8]. It has also been shown to decrease skin reactivity to irritants such as sodium lauryl sulfate [13]. Furthermore, the addition of 5\% urea to a hydroalcoholic gel reduced TEWL, dryness and skin reactivity [14]. Regulatory properties of urea at the epidermis could even help to reduce skin aging [15].

Choosing an emollient/moisturizer among the vast variety of available products can be challenging. The ideal emollient/moisturizer should reduce TEWL, restore the skin barrier function, be hypoallergenic, non-comedogenic and fragrance-free, be easily absorbed and have an acceptable cost [10]. Urea-containing

Table 2 Urea concentration, effects and indications

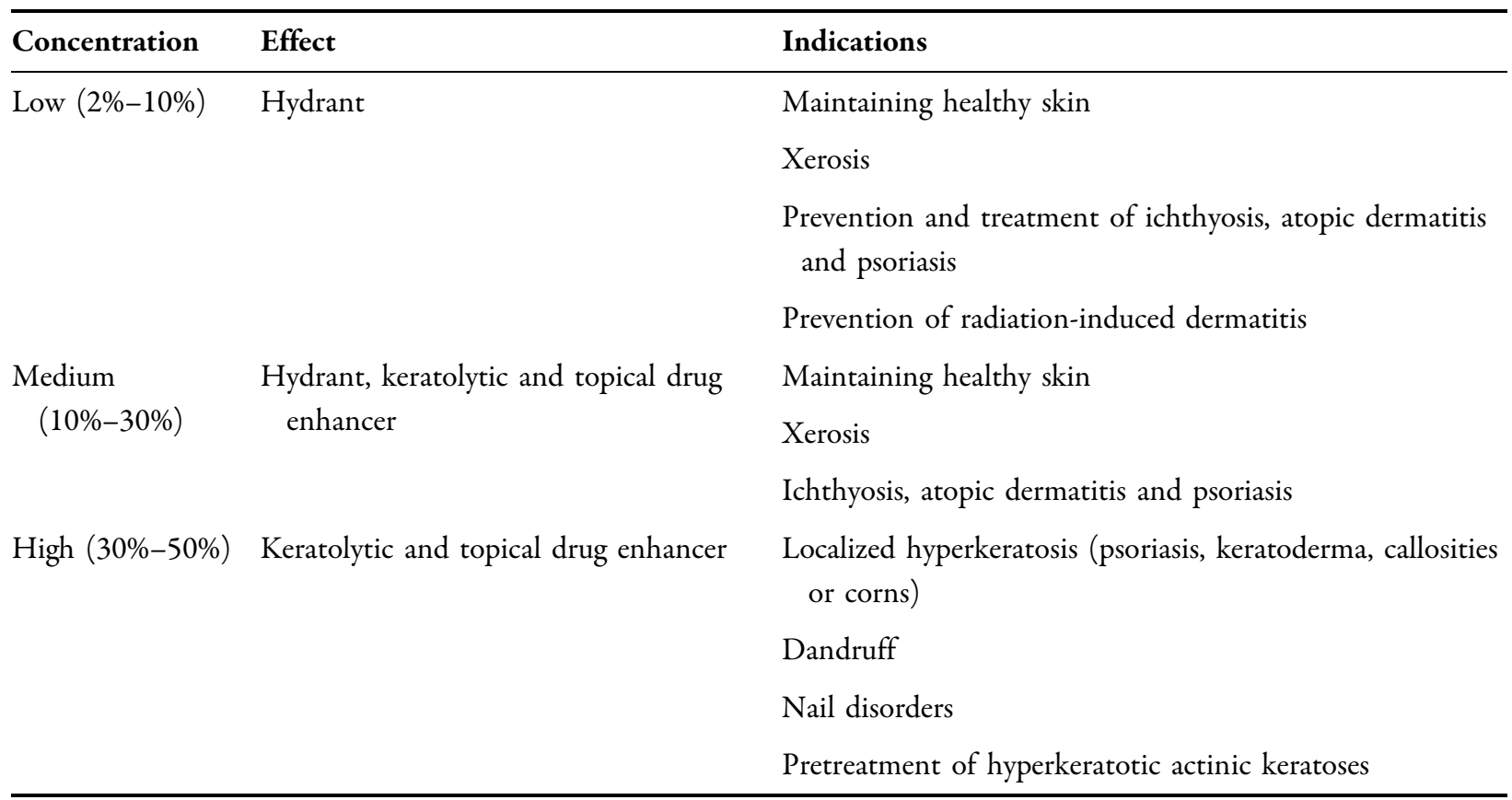


formulations can potentially offer these characteristics and be indicated for maintaining healthy skin and for treating a wide range of skin disorders, as discussed in the following sections.

\section{Atopic Dermatitis}

The skin of patients with $\mathrm{AD}$ is characterized by increased TEWL and dysfunction of the skin barrier [16]. The use of emollient and moisturizing creams is considered a cornerstone of the management of this disorder [16, 17]. The impact of moisturizers on $\mathrm{AD}$ management has been documented in a large number of clinical trials of varying quality and design [18]. However, given the wide variety of products available, choosing an emollient/moisturizer can be difficult, and some products may even worsen $\mathrm{AD}$ or induce contact dermatitis [16, 18].

Urea-containing preparations have been shown to decrease TEWL and improve hydration of the stratum corneum and water-binding capacity in eczematous skin [3, 11, 13, 19]. In a murine model of $\mathrm{AD}$, a urea-based formulation improved the antimicrobial defense and the barrier function of the skin by regulating genes involved in the production of antimicrobial peptides, differentiation of keratinocytes and lipid synthesis [8]. The use of topical urea at concentrations ranging from $4 \%$ to $20 \%$ has been documented in multiple clinical trials [18-25]. A systematic review of the use of emollients in AD found that clinical effectiveness appears most well documented for ureabased preparations and recommends topical urea as a first-line choice in $\mathrm{AD}$ [18]. A randomized clinical trial (RCT) demonstrated the superiority of urea $4 \%$ versus glycerin $20 \%$ in reducing TEWL in patients with AD [19]. An RCT comparing two urea-based formulations (5\% urea moisturizer vs 10\% urea lotion) found no significant differences between them in reducing the Scoring Atopic Dermatitis (SCORAD) severity index [22].

Regarding prevention of $\mathrm{AD}$ relapse, an RCT including $172 \mathrm{AD}$ patients showed that prolonged use of a preparation containing 5\% urea significantly increased the eczema-free time, reducing the risk of relapse by $37 \%$ [21]. Similar findings were reported in another RCT on 53 individuals with hand eczema [20].

\section{Xerosis}

Multiple clinical studies support the use of ureacontaining formulations at varying concentrations (most frequently 10\%) for the treatment of xerosis in individuals with or without skin disorders [3, 6]. A controlled clinical study of 12 patients presenting with severe dry skin on the inferior extremities showed a significant reduction in TEWL after twice-daily application of a $15 \%$ urea cream [26]. A placebo-controlled, double-blind study of 21 healthy individuals showed that a once-daily application of a $20 \%$ urea-containing cream led to a significant reduction of TEWL [8]. An experimental study of five healthy subjects has demonstrated that a $20 \%$ urea-based cream significantly increased the water content of the stratum corneum [27].

Xerosis in the elderly: Xerosis can affect up to $75 \%$ of older adults. In a prospective study of 42 individuals $>60$ years old, application of a $5 \%$ urea-containing cream showed increased skin hydration compared to a control emollient [28]. In an experimental study of ten patients with senile xerosis, the application of a $10 \%$ ureabased cream led to marked clinical improvement [29].

Xerosis in patients with diabetes mellitus: Xerosis is frequently observed in diabetic patients and can increase the risk of skin infection and ulceration. In an RCT of 40 individuals with type 2 diabetes mellitus, a 5\% urea-based formulation with other ingredients such as arginine and carnosine increased skin hydration and significantly decreased xerosis compared to a glycerol-based emollient [30]. In another RCT of 40 patients with diabetes $>60$ years a moisturizer containing $10 \%$ urea provided faster and better clinical improvement of xerosis of the feet than a control emollient [31]. A doubleblind pilot study of 30 diabetic individuals compared the efficacy of a cream containing $10 \%$ urea and a cream containing $25 \%$ urea in the treatment of anhydrosis in the diabetic foot. Although both urea creams induced a 
significant increase in skin hydration, the 25\% urea cream produced better results [32].

Xerosis in hemodialyzed patients: Xerosis is very common among patients undergoing hemodialysis. Associated factors for the development of xerosis in these individuals include accumulation of uremic toxins, presence of comorbidities such as diabetes mellitus and viral hepatitis, and systemic inflammation, among others. An RCT on 15 hemodialysis patients with severe xerosis showed that the use of a lotion containing 10\% urea and dexpanthenol led to significant clinical improvement and reduction in pruritus [33].

Foot xerosis: Xerosis of the feet is a very common condition. A systematic review of the literature revealed that emollients containing urea have been the most researched [34]. Fourteen studies using urea in varying concentration (5\%-40\%) have shown good clinical responses [34].

\section{Ichthyosis}

Ichthyoses are a group of genetic disorders characterized by cutaneous xerosis. Ichthyosis vulgaris (IV) is the most prevalent subtype. More than a dozen clinical trials carried out on patients with diverse ichthyoses, using ureabased formulations at concentrations ranging from $2 \%$ to $10 \%$ (10\% has been the most frequently used), have shown significant improvement of clinical parameters together with increased water retention capacity of the stratum corneum $[3,11]$. In an RCT on 30 IV patients, a $10 \%$ urea formulation demonstrated a greater reduction in flaking, roughness, redness and cracking compared to a glycerol-based emollient cream [35]. In a double-blind clinical trial on $84 \mathrm{IV}$ individuals, a urea-based cream was significantly better in controlling the ichthyosis than $2 \%$ salicylic acid ointment and paraffin [36]. In a prospective study on five patients with IV, a $10 \%$ urea-containing emulsion significantly decreased pruritus and xerosis in all individuals. Videodermatoscopy and confocal reflectance microscopy were performed, revealing a marked reduction in desquamation and normalization of furrow size and morphology [37]. In patients with IV, urea can not only increase stratum corneum hydration, but may also upregulate filaggrin gene expression [11, 37].

\section{Psoriasis}

Urea-based formulations can improve hydration of the stratum corneum and its water retention capacity and decrease TEWL, pruritus, desquamation and hyperkeratosis in patients with psoriasis $[3,4,12,38]$. High urea concentration (40\%-50\%) can be an excellent alternative in the treatment of localized hyperkeratotic plaques of psoriasis [4]. Urea at $40 \%$ together with bifonazole $1 \%$ can be effective in the management of scalp psoriasis [3]. Medium urea concentration (17\%) together with $0.1 \%$ dithranol has shown good clinical responses [3]. In an RCT on 70 patients with scalp psoriasis, the use of a urea-based shampoo significantly reduced itching and psoriasis severity index scores [39]. While medium or high urea concentration is usually indicated in psoriasis, some studies support the use of low urea concentration (5\% to $12 \%$ ) [12]. Urea at low concentration can also increase the effectiveness of topical betamethasone and calcipotriol [11, 38].

\section{Hyperkeratosis and/or Palmoplantar Keratodermas}

Palmoplantar keratodermas are a broad spectrum of acquired and hereditary disorders characterized by abnormal thickening of the skin on the palms and soles. Urea at $>30 \%$ concentration may be a good alternative for symptomatic treatment $[12,40]$. Urea can be indicated together with topical salicylic acid, lactic acid, retinoids and corticosteroids.

Targeted therapy has revolutionized cancer therapy, and a wide range of dermatologic adverse events has been described. Hand-foot skin reaction (HFSR) is characterized by painful hyperkeratotic lesions on the palms and soles and can severely affect health-related quality of life and lead to discontinuation or drug dose reduction. An RCT on 871 patients with advanced hepatocellular carcinoma treated 
with sorafenib showed that prophylactic treatment with a $10 \%$ urea-based cream can significantly reduce HFSR rates, extend the time to its first occurrence and improve patient quality of life [41]. Similar findings were reported in a recent RCT on 288 patients under sorafenib therapy [42].

\section{Irradiation-Induced Dermatitis}

Radiation-induced dermatitis is one of the most common side effects of radiation therapy. In a prospective study of 98 patients with breast cancer, intensive use of a lotion containing 3\% urea, polidocanol and hyaluronic acid doubled the probability of not developing radiodermatitis during radiotherapy, and intensive use was more effective than standard use in reducing the incidence of skin toxicity and skin toxicity grade 2 or higher [43].

\section{Seborrheic Dermatitis of the Scalp}

The most frequently prescribed pharmacological treatments for seborrheic dermatitis are antifungals and corticosteroids. Urea has antifungal and antimicrobial properties together with high moisturizing capacity and a keratolytic effect. The use of keratolytics can prevent scaling or crusting in patients with seborrheic dermatitis and can increase the penetration of topical drugs [44]. In a clinical study of patients with mild-moderate seborrheic dermatitis, the use of a shampoo containing urea, glycolic acid, salicylic acid, pale ichthyol and laureth significantly reduced irritation and itching and improved hair appearance [40]. The use of a formulation containing $40 \%$ urea and $1 \%$ bifonazole may be an option in severe cases [3].

\section{Actinic Keratosis and Cancerization Field}

Treatment of hyperkeratotic actinic keratoses can be challenging. Pretreatment with keratolytics such as urea-based formulations at high concentration (40\%), salicylic acid or topical retinoids can increase response rates [45].

\section{Nail Disorders}

Urea at $40 \%-50 \%$ has been extensively used for the treatment of nail disorders; it can enhance nail permeability and soften the nail plate [4]. Urea at medium or high concentrations can improve the efficacy of topical antifungal drugs by increasing penetration and biodisponibility $[4,6,12]$. A recent systematic review showed that $40 \%$ urea plus topical antifungals can induce clinical improvement in $29 \%-85 \%$ of cases [46].

Urea at high concentration can be an excellent alternative in the management of nail psoriasis, onychogryphosis and pincer nails, among others, as it can allow cutting and proper care of thickened nails, reducing patient discomfort, with minimal associated adverse events. The application of urea under occlusion leads to faster clinical responses [4].

\section{Tinea Pedis}

Tinea pedis can take a chronic and recalcitrant course. Urea has antimicrobial properties and reduces flaking and cracks. The use of urea at low concentration (10\%) together with topical antifungals can reduce treatment duration and improve clinical response [2]. Urea at high concentration can be useful in the management of hyperkeratotic tinea pedis.

\section{CONCLUSIONS}

Urea plays a fundamental role in regulating keratinocyte proliferation, the skin's barrier function and antimicrobial defense. Urea-based formulations are well tolerated and one of the most commonly prescribed moisturizers and keratolytic agents for dermatologic patients. Urea-containing formulations can be indicated to maintain healthy skin and treat a wide range of skin disorders such as $\mathrm{AD}$, xerosis, psoriasis, ichthyosis and seborrheic dermatitis, among many others, and to improve penetration of topical drugs into the skin and nails.

The effects of urea on gene expression predict, in the near future, a relevant role for urea- 
containing formulations for skin barrier therapy.

\section{ACKNOWLEDGMENTS}

Funding. The journal's Rapid Service Fee was supported by ISDIN.

Authorship. All named authors meet the International Committee of Medical Journal Editors (ICMJE) criteria for authorship for this article, take responsibility for the integrity of the work as a whole, and have given their approval for this version to be published.

Author Contributions. Jaime PiqueroCasals, Daniel Morgado-Carrasco, Corinne Granger and Jean Krutmann contributed equally to concept and design of the study. Jaime Piquero-Casals, Daniel Morgado-Carrasco, Corinne Granger, Carles Trullàs, América Jesús-Silva and Jean Krutmann contributed to the draft and revised the manuscript.

Disclosures. Jaime Piquero-Casals, Daniel Morgado-Carrasco, and Jean Krutmann have received consultant fees from ISDIN. Corinne Granger and Carles Trullàs are employees of ISDIN. América Jesús-Silva has nothing to disclose.

Compliance with Ethics Guidelines. This article is based on previously conducted studies and does not contain any studies with human participants or animals performed by any of the authors.

Data Availability. Data sharing is not applicable to this article as no datasets were generated or analyzed during the current study.

Open Access. This article is licensed under a Creative Commons Attribution-NonCommercial 4.0 International License, which permits any non-commercial use, sharing, adaptation, distribution and reproduction in any medium or format, as long as you give appropriate credit to the original author(s) and the source, provide a link to the Creative Commons licence, and indicate if changes were made. The images or other third party material in this article are included in the article's Creative Commons licence, unless indicated otherwise in a credit line to the material. If material is not included in the article's Creative Commons licence and your intended use is not permitted by statutory regulation or exceeds the permitted use, you will need to obtain permission directly from the copyright holder. To view a copy of this licence, visit http://creativecommons.org/licenses/by$\mathrm{nc} / 4.0 /$.

\section{REFERENCES}

1. Friedman AJ, von Grote EC, Meckfessel MH. Urea: a clinically oriented overview from bench to bedside. J Drugs Dermatol. 2016;15:633-9.

2. Verzì AE, Musumeci ML, Lacarrubba F, Micali G. History of urea as a dermatological agent in clinical practice. Int J Clin Pract. 2020;74(Suppl 187): e13621.

3. Pan M, Heinecke G, Bernardo S, et al. Urea: a comprehensive review of the clinical literature. Dermatol Online J. 2013;19:20392.

4. Starace M, Alessandrini A, Piraccini BM. Clinical evidences of urea at high concentration on skin and annexes. Int J Clin Pract. 2020;74(Suppl 187): e13740.

5. Piraccini BM, Alessandrini A, Bruni F, Starace M. Acute periungueal dermatitis induced by application of urea-containing cream under occlusion. J Dermatol Case Rep. 2012;6:18-20.

6. Celleno L. Topical urea in skincare: a review. Dermatol Ther. 2018;31:12690.

7. Trullàs-Cabanes C, Mirada-Ferré A, Salomon-Niera $\mathrm{M}$. El valor de la urea en el cuidado y tratamiento de la piel. Revista Dermatológica Perú. 2008;18(1): 41-4.

8. Grether-Beck S, Felsner I, Brenden $H$, et al. Urea uptake enhances barrier function and antimicrobial defense in humans by regulating epidermal gene expression. J Invest Dermatol. 2012;132:1561-72.

9. Berardesca E, Cameli N. Non-invasive assessment of urea efficacy: a review. Int J Clin Pract. 2020;74(Suppl 187):e13603. 
10. Sethi A, Kaur T, Malhotra S, Gambhir M. Moisturizers: the slippery road. Indian J Dermatol. 2016;61: 279-87.

11. Lacarrubba F, Nasca MR, Puglisi DF, Micali G. Clinical evidences of urea at low concentration. Int J Clin Pract. 2020;74(Suppl 187):e13626.

12. Dall'Oglio F, Tedeschi A, Verzì AE, et al. Clinical evidences of urea at medium concentration. Int $\mathrm{J}$ Clin Pract. 2020;74:e13815.

13. Loden M. Urea-containing moisturizers influence barrier properties of normal skin. Arch Dermatol Res. 1996;288:103-7.

14. Lodén M. Ethanol-based disinfectants containing urea may reduce soap sensitivity. Dermatitis. 2020;31:328-32.

15. Sanz MT, Campos C, Milani M, et al. Biorevitalizing effect of a novel facial serum containing apple stem cell extract, pro-collagen lipopeptide, creatine, and urea on skin aging signs. J Cosmet Dermatol. 2016;15:24-30.

16. Wollenberg A, Christen-Zäch S, Taieb A, et al. ETFAD/EADV Eczema task force 2020 position paper on diagnosis and treatment of atopic dermatitis in adults and children. J Eur Acad Dermatol Venereol. 2020;34:2717-44.

17. Garnacho-Saucedo G, Salido-Vallejo R, MorenoGiménez JC. Atopic dermatitis: update and proposed management algorithm. Actas Dermosifiliogr. 2013;104:4-16.

18. Lindh JD, Bradley M. Clinical effectiveness of moisturizers in atopic dermatitis and related disorders: a systematic review. Am J Clin Dermatol. 2015;16:341-59.

19. Lodén M, Andersson AC, Andersson C, et al. Instrumental and dermatologist evaluation of the effect of glycerine and urea on dry skin in atopic dermatitis. Skin Res Technol. 2001;7:209-13.

20. Lodén M, Wirén K, Smerud K, et al. Treatment with a barrier-strengthening moisturizer prevents relapse of hand-eczema. An open, randomized, prospective, parallel group study. Acta Derm Venereol. 2010;90:602-6.

21. Åkerström U, Reitamo S, Langeland T, et al. Comparison of moisturizing creams for the prevention of atopic dermatitis relapse: a randomized doubleblind controlled multicentre clinical trial. Acta Derm Venereol. 2015;95:587-92.

22. Bissonnette R, Maari C, Provost N, et al. A doubleblind study of tolerance and efficacy of a new urea- containing moisturizer in patients with atopic dermatitis. J Cosmet Dermatol. 2010;9:16-21.

23. Lodén M, Andersson A-C, Anderson C, et al. A double-blind study comparing the effect of glycerin and urea on dry, eczematous skin in atopic patients. Acta Derm Venereol. 2002;82:45-7.

24. Hagströmer L, Nyrén M, Emtestam L. Do urea and sodium chloride together increase the efficacy of moisturisers for atopic dermatitis skin? A comparative, double-blind and randomised study. Skin Pharmacol Appl Skin Physiol. 2001;14:27-33.

25. Nasrollahi SA, Ayatollahi A, Yazdanparast T, et al. Comparison of linoleic acid-containing water-in-oil emulsion with urea-containing water-in-oil emulsion in the treatment of atopic dermatitis: a randomized clinical trial. Clin Cosmet Investig Dermatol. 2018;11:21-8.

26. Rosado C, Pinto P, Rodrigues LM. Assessment of moisturizers and barrier function restoration using dynamic methods. Skin Res Technol. 2009;15: 77-83.

27. Egawa M. In vivo simultaneous measurement of urea and water in the human stratum corneum by diffuse-reflectance near-infrared spectroscopy. Skin Res Technol. 2009;15:195-9.

28. Danby SG, Brown K, Higgs-Bayliss T, et al. The effect of an emollient containing urea, ceramide $\mathrm{NP}$, and lactate on skin barrier structure and function in older people with dry skin. Skin Pharmacol Physiol. 2016;29:135-47.

29. Horii I, Nakayama Y, Obata M, Tagami H. Stratum corneum hydration and amino acid content in xerotic skin. Br J Dermatol. 1989;121:587-92.

30. Federici A, Federici G, Milani M. An urea, arginine and carnosine based cream (Ureadin Rx Db ISDIN) shows greater efficacy in the treatment of severe xerosis of the feet in Type 2 diabetic patients in comparison with glycerol-based emollient cream. A randomized, assessor-blinded, controlled trial. BMC Dermatol. 2012;12:16.

31. Pham HT, Exelbert L, Segal-Owens AC, Veves A. A prospective, randomized, controlled double-blind study of a moisturizer for xerosis of the feet in patients with diabetes. Ostomy Wound Manage. 2002;48:30-6.

32. Baird SA, Skinner CM, Trail S, Frankis JS. Anhydrosis in the diabetic foot: a comparison of two urea creams. Diabetic Foot J. 2003;6:4.

33. Castello M, Milani M. Efficacy of topical hydrating and emollient lotion containing $10 \%$ urea ISDIN® plus dexpanthenol (Ureadin $\mathrm{Rx}$ 10) in the 
treatment of skin xerosis and pruritus in hemodialyzed patients: an open prospective pilot trial. G Ital Dermatol Venereol. 2011;146:321-5.

34. Parker J, Scharfbillig R, Jones S. Moisturisers for the treatment of foot xerosis: a systematic review. J Foot Ankle Res. 2017;10:9.

35. Tadini G, Giustini S, Milani M. Efficacy of topical $10 \%$ urea-based lotion in patients with ichthyosis vulgaris: a two-center, randomized, controlled, single-blind, right-vs.-left study in comparison with standard glycerol-based emollient cream. Curr Med Res Opin. 2011;27:2279-84.

36. Pope FM, Rees JK, Wells RS, Lewis KG. Out-patient treatment of ichthyosis: a double-blind trial of ointments. Br J Dermatol. 1972;86:291-6.

37. Benintende C, Boscaglia S, Dinotta F, et al. Treatment of ichthyosis vulgaris with a urea-based emulsion: videodermatoscopy and confocal microscopy evaluation. G Ital Dermatol Venereol. 2017;152:555-9.

38. Jacobi A, Mayer A, Augustin M. Keratolytics and emollients and their role in the therapy of psoriasis: a systematic review. Dermatol Ther (Heidelb). 2015;5:1-18.

39. Rossi A, Pranteda G, Iorio A, et al. Efficacy of Iralfaris shampoo in the treatment of scalp psoriasis: a videodermoscopy evaluation prospective study in 70 patients. G Ital Dermatol Venereol. 2012;147: 625-30.

40. Piraccini BM, Brandi N, Alessandrini A, et al. Efficacious and safe management of thick scales, redness and flaky scalp condition using a specific shampoo containing urea, glycolic acid, salicylic acid, icthyol pale and laureth 9. null. 2018;5: 1475095.

41. Ren Z, Zhu K, Kang H, et al. Randomized controlled trial of the prophylactic effect of urea-based cream on sorafenib-associated hand-foot skin reactions in patients with advanced hepatocellular carcinoma. J Clin Oncol. 2015;33:894-900.

42. Lee Y-S, Jung YK, Kim JH, et al. Effect of urea cream on sorafenib-associated hand-foot skin reaction in patients with hepatocellular carcinoma: a multicenter, randomised, double-blind controlled study. Eur J Cancer. 2020;140:19-27.

43. Pardo Masferrer J, Murcia Mejía M, Vidal Fernández $\mathrm{M}$, et al. Prophylaxis with a cream containing urea reduces the incidence and severity of radio-induced dermatitis. Clin Transl Oncol. 2010;12:43-8.

44. Piquero-Casals J, Hexsel D, Mir-Bonafé JF, RozasMuñoz E. Topical non-pharmacological treatment for facial seborrheic dermatitis. Dermatol Ther (Heidelb). 2019;9:469-77.

45. Piquero-Casals J, Morgado-Carrasco D, Gilaberte Y, et al. Management pearls on the treatment of actinic keratoses and field cancerization. Dermatol Ther (Heidelb). 2020. https://doi.org/10.1007/ s13555-020-00425-4.

46. Dars S, Banwell HA, Matricciani L. The use of urea for the treatment of onychomycosis: a systematic review. J Foot Ankle Res. 2019;12:22. 